\title{
Setting the Stage for 2018: How the Changes in the American Joint Committee on Cancer/Union for International Cancer Control Cancer Staging Manual Eighth Edition Impact Radiologists
}

\author{
(D)C.M. Glastonbury, DS.K. Mukherji, (DB. O'Sullivan, and (D)W.M. Lydiatt
}

\begin{abstract}
SUMMARY: The updated eighth edition of the Cancer Staging Manual of the American Joint Committee on Cancer will be implemented in January 2018. There are multiple changes to the head and neck section of the manual, which will be relevant to radiologists participating in multidisciplinary head and neck tumor boards and reading pretreatment head and neck cancer scans. Human papillomavirus-related/ p16(+) oropharyngeal squamous cell carcinoma will now be staged separately; this change reflects the markedly better prognosis of these tumors compared with non-human papillomavirus/p16(-) oropharyngeal squamous cell carcinoma. Nodal staging has dramatically changed so that there are different tables for human papillomavirus/p16(+) oropharyngeal squamous cell carcinoma, Epstein-Barr virusrelated nasopharyngeal carcinoma, and all other head and neck squamous cell carcinomas. Extranodal extension of tumor is a new clinical feature for this third staging group. In the oral cavity, the pathologically determined depth of tumor invasion is a new staging criterion, while extrinsic tongue muscle invasion is no longer part of staging. This review serves to educate radiologists on the eighth edition changes and their rationale.
\end{abstract}

ABBREVIATIONS: $\mathrm{AJCC}=$ American Joint Committee on Cancer; $\mathrm{CN}=$ clinical nodal staging; $\mathrm{DOI}=$ depth of invasion; $\mathrm{EBV}=\mathrm{Epstein-Barr}$ virus; $\mathrm{ENE}=$ extranodal extension; $\mathrm{HN}=$ head and neck; HPV = human papillomavirus; NPC = nasopharyngeal carcinoma; OPSCC = oropharyngeal squamous cell carcinoma; $\mathrm{pN}=$ pathologic nodal staging; SCC = squamous cell carcinoma; TNM = tumor, node, metastasis; UICC = Union for International Cancer Control

$\mathbf{T}$ he tumor, node, metastasis (TNM) cancer staging system is a collaborative effort between the American Joint Committee on Cancer (AJCC) and the Union for International Cancer Control (UICC) to systematically record, analyze, and better understand cancer and cancer treatment worldwide. ${ }^{1,2}$ For the clinical team, this staging system allows a common, consistent nomenclature and provides a framework for managing patient care. For the individual patient, this system allows classification of the extent of disease so that prognostic information can be provided and treatment options determined.

Received July 5, 2017; accepted after revision August 17.

From the Department of Radiology and Biomedical Imaging (C.M.G.), University of California, San Francisco, California; Department of Radiology (S.K.M.), Michigan State University, East Lansing, Michigan; Department of Radiation Oncology (B.O.), Princess Margaret Cancer Center, University of Toronto, Toronto, Ontario, Canada; Department of Surgery, Head and Neck Oncology (W.M.L.), Estabrook Cancer Center, Nebraska Methodist Hospital, Omaha, Nebraska; Creighton University (W.M.L.), Omaha, Nebraska; and Naval Medical Center (W.M.L.), Portsmouth, Virginia.

All authors are members of the Head and Neck Expert Panel for the eighth edition of the American Joint Committee on Cancer Cancer Staging Manual. W.M. Lydiatt is the Panel Vice-Chair. B. O'Sullivan is the UICC representative and the chair of the UICC HN subcommittee.

Please address correspondence to Christine M. Glastonbury, MBBS, Department of Radiology, Box 0628, Room L-358, 505 Parnassus Ave, San Francisco, CA 94143-0628; e-mail: christine.glastonbury@ucsf.edu; @CMGlastonbury

http://dx.doi.org/10.3174/ajnr.A5409
Periodically, the TNM system is revised to reflect changes in our understanding of tumor pathology and pathophysiology. The AJCC Cancer Staging Manual seventh edition was implemented in 2010, with the most recent eighth edition completed and published this year and implemented for staging of all patients with cancer as of January 1, 2018. ${ }^{3}$ There are many important changes to the head and neck (HN) section, which again forms Part II of the AJCC eighth edition and has expanded to 11 chapters. Two new chapter titles have been added to the head and neck section, one on cervical nodes and unknown primary tumors and one on $\mathrm{HN}$-specific cutaneous malignancy. Pharynx cancer has been divided into 3 separate chapters: "Nasopharynx," "Human Papillomavirus (HPV)-Mediated p16+ Oropharyngeal Cancer," and "p16- plus Hypopharynx." Thyroid cancer staging has been moved from the HN section to the "Endocrine System" (Part XVII), and orbital and eyelid tumors remain in "Ophthalmic Sites" (Part XV). Within the HN chapters, there are important changes related to extranodal extension (ENE) of tumor and some key changes to T-categories, some of which relate to pathology staging and others to imaging. Changes to the eighth edition of the AJCC Cancer Staging Manual head and neck section require knowing specific clinical and/or pathologic information in order to use the correct staging table. Specifically, it is important to know the p16 status of oropharynx primary tumors because 
Table 1: Clinical N staging for all non-HPV, non-EBV SCCs ${ }^{a}$

\begin{tabular}{|c|c|}
\hline N Category & N Criteria \\
\hline $\mathrm{Nx}$ & Regional lymph nodes cannot be assessed \\
\hline No & No regional lymph node metastasis \\
\hline N1 & $\begin{array}{l}\text { Metastasis in a single ipsilateral lymph node } \leq 3 \mathrm{~cm} \text { in greatest dimension } \\
\text { and ENE- }\end{array}$ \\
\hline $\mathrm{N} 2 \mathrm{a}$ & $\begin{array}{l}\text { Metastasis in a single ipsilateral lymph node }>3 \mathrm{~cm} \text { but } \leq 6 \mathrm{~cm} \text { in greatest } \\
\text { dimension and ENE- }\end{array}$ \\
\hline $\mathrm{N} 2 \mathrm{~b}$ & $\begin{array}{l}\text { Metastasis in multiple ipsilateral nodes, none }>6 \mathrm{~cm} \text { in greatest dimension } \\
\text { and ENE- }\end{array}$ \\
\hline N2c & $\begin{array}{l}\text { Metastasis in bilateral or contralateral lymph node(s), none }>6 \mathrm{~cm} \text { in greatest } \\
\text { dimension and ENE- }\end{array}$ \\
\hline N3a & Metastasis in a lymph node $>6 \mathrm{~cm}$ in greatest dimension and ENE- \\
\hline N3b & Metastasis in any node(s) with clinically overt ENE+ (ENEc) \\
\hline
\end{tabular}

Note:-ENEc indicates invasion of skin, infiltration of musculature, dense tethering or fixation to adjacent structures, or cranial nerve, brachial plexus, sympathetic trunk, or phrenic nerve invasion with dysfunction.

a Note the addition of ENE to the staging system, creating a new N3b designation in the eighth edition of the AJCC Cancer Staging Manual. Table used with the permission of the American Joint Committee on Cancer (AJCC), Chicago, Illinois. The original source for this material is the AJCC Cancer Staging Manual.'

Table 2: Clinical nodal staging for HPV-related OPSCC ${ }^{a}$

\begin{tabular}{ll}
\hline N Category & \multicolumn{1}{c}{ N Criteria } \\
\hline Nx & Regional lymph nodes cannot be assessed \\
N0 & No regional lymph node metastasis \\
N1 & One or more ipsilateral lymph nodes, none larger \\
& than $6 \mathrm{~cm}$ \\
N2 & Contralateral or bilateral lymph nodes, none $>6 \mathrm{~cm}$ \\
N3 & Lymph node $(\mathrm{s})>6 \mathrm{~cm}$ \\
\hline
\end{tabular}

a The HPV-related OPSCC nodal staging shows a dramatic change compared with the eighth edition non-HPV OPSCC staging. Table adapted with permission of the American Joint Committee on Cancer, Chicago, Illinois. The original source for this material is the AJCC Cancer Staging Manual.'

p16(+) and p16(-) oropharynx tumors are staged differently. Also, when evaluating cases of nodal disease, one must know whether an HN primary tumor is known and whether that tumor is a skin or mucosal primary. This review serves to summarize the critical changes relevant to all imagers reading HN CT, MR imaging, or PET/CT scans, particularly those participating in tumor board clinical staging.

\section{AJCC Changes with Impact for the Radiology Report}

Cervical Nodes and Extranodal Extension. The changes made to nodal staging might be perceived by radiologists as the most confusing part of the $\mathrm{HN}$ section in the eighth edition, and while many areas elsewhere in the $\mathrm{HN}$ section are about simplification from the seventh edition, nodal definition shows increasing complexity. This reflects the key changes resulting from a better understanding of both HPV-related oropharyngeal squamous cell carcinoma (OPSCC) and the significance of extranodal extension of tumor in non-HPV and non-Epstein-Barr virus (EBV) nodal disease. $^{4-11}$

There are now 5 different staging tables for nodes, 2 of which are purely pathologic staging $(\mathrm{pN})$ following cervical lymph node dissection (of $\geq 15$ nodes) (Tables $1-3$ ):

- HPV-related OPSCC: clinical (cN) and separate pathologic $(\mathrm{pN})$ nodal staging

- EBV-related nasopharyngeal carcinoma (NPC): clinical staging very similar to that in the seventh edition with changes to N3 designation only

- All other HN squamous cell carcinomas (SCCs): clinical and separate pathologic nodal staging.
Particularly given this increasing complexity, referring to the published charts is the best way to avoid confusion and errors in staging. For radiologists reading scans who are unsure of the HPV or EBV tumor status, it will not be possible to know which clinical nodal category $(\mathrm{cN})$ table to use. In the first instance, radiologists should, if possible, search the pathology report in the electronic medical record for this information. When EBV/HPV/p16 information is not available, it is important that the nodal level system be used to describe the location of ipsilateral and/or contralateral abnormal nodes to enable the clinician to assign a $\mathrm{cN}$. When one determines the presence of concerning adenopathy, all morphologic characteristics of a node should be assessed, particularly in the expected drainage pattern of the known primary tumor: size (>10-mm short axis), shape (rounded), loss of fatty hilus, focal nodal inhomogeneity (focal necrosis), and cystic change. Additionally, for all tumors except nasopharyngeal carcinoma and HPV/ p16(+) oropharyngeal tumors, radiologists should evaluate evidence of extranodal extension of the tumor. Currently, the clinical designation of ENE + requires overt clinical examination evidence of ENE, such as skin invasion, infiltration of muscle with tethering or fixation to adjacent structures, or large-nerve invasion with dysfunction such as the brachial plexus, sympathetic trunk, phrenic, or cranial nerves. While indistinct nodal margins and an irregular nodal capsular enhancement suggest ENE, the strongest imaging feature supporting the clinical diagnosis of ENE is clear infiltration of perinodal tumor into adjacent fat or muscle. ${ }^{12-14}$ It will be most useful for radiologists to describe features suspicious for ENE so that the clinician can re-evaluate the clinical nodal status. Improving the imaging specificity for ENE is clearly an area of focus for radiologists and therefore potentially contributing to future revisions of the AJCC Cancer Staging Manual.

HPV-Related (p16+) Oropharyngeal Squamous Cell Carcinoma. Probably the most significant change to HN tumor staging is the separation of tumors related to human papilloma virus infection and/or determined to be $\mathrm{p} 16+$ on immunohistochemistry. This separate staging designation reflects the novelty of this disease, which exhibits an overall markedly better prognosis compared with p16- and largely tobacco- and alcohol-related OPSCC. ${ }^{4-7,11}$

Metastatic nodes with HPV-related OPSCC are frequently large, multiple, and/or bilateral, yet the disease will overall have a much better survival than non-HPV OPSCC with similar extent of nodal involvement. Thus, there have been significant changes made to the nodal staging, which will result in quite dramatic changes to overall prognostic grouping compared with the staging used for all OPSCC in the seventh edition (Figs 1 and 2 and Tables 2, 4, and 5). For clinical N categories of HPV/p16+ OPSCC, any ipsilateral node or nodes that are $\leq 6 \mathrm{~cm}$ are designated N1 disease. Bilateral or contralateral nodes of $\leq 6 \mathrm{~cm}$ are designated N2 disease, and N3 disease is determined when a nodal mass is $>6$ 


\begin{tabular}{ll} 
N Category & N Criteria \\
\hline $\mathrm{N} x$ & Regional lymph nodes cannot be assessed \\
N0 & No regional lymph node metastasis \\
N1 & Unilateral metastasis in cervical lymph node $(\mathrm{s})$ and/or unilateral or bilateral metastasis in retropharyngeal lymph \\
& node $(\mathrm{s}) \leq 6 \mathrm{~cm}$ in greatest dimension, above the caudal border of cricoid cartilage \\
$\mathrm{N} 2$ & Bilateral metastasis in cervical lymph nodes $\leq 6 \mathrm{~cm}$ in greatest dimension, above the caudal border of cricoid cartilage \\
$\mathrm{N} 3$ & Unilateral or bilateral metastasis in cervical lymph node $(\mathrm{s})>6 \mathrm{~cm}$ in greatest dimension, and/or extension below the \\
& caudal border of cricoid cartilage \\
\hline
\end{tabular}

${ }^{a}$ Changes in the eighth edition reflect the designation of level IV or VB nodes as N3 disease with no distinct a or b status. Table adapted with permission of the American Joint Committee on Cancer, Chicago, Illinois. The original source for this material is the AJCC Cancer Staging Manual.'

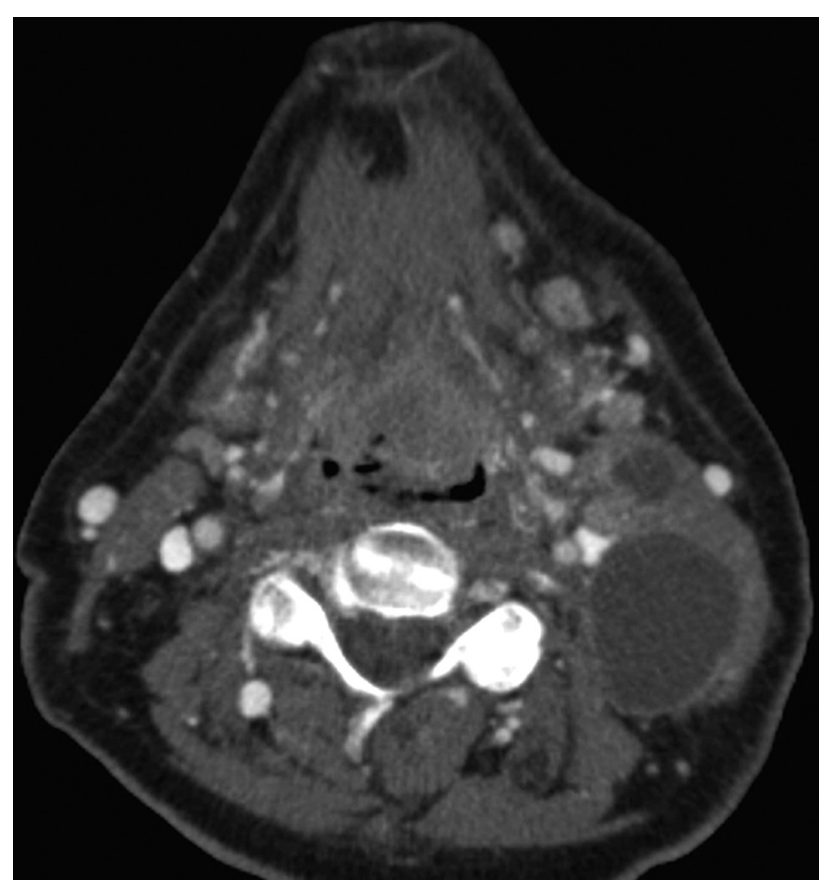

FIG 1. Axial contrast-enhanced CT in a 69-year-old man demonstrates a heterogeneously enlarged lingual tonsil filling the valleculae and at least 2 left cystic cervical nodes. No right neck adenopathy was evident. Biopsy of the primary site revealed p16(+) OPSCC. This was staged as CT2N2b, stage IVA OPSCC. In the eighth edition of the AJCC, the T-category does not change; however, multiple ipsilateral nodes are now assigned $\mathrm{N} 1$ category if $<6 \mathrm{~cm}$ in greatest diameter. In the eighth edition, T2N1 p16(+) OPSCC is designated stage I disease.

$\mathrm{cm}$. For prognostic staging of tumors with $\mathrm{T} 0-2, \mathrm{~N} 1$ disease is stage I, N2 is stage II, and N3 is stage III. For T3 tumors, stage II is a minimum stage regardless of $\mathrm{N}$, and for $\mathrm{T} 4$ or N3, stage III is the minimum as seen in Table 5. Stage IV will now be reserved for M1 disease, regardless of $\mathrm{T}$ and $\mathrm{N}$. There are no changes to the primary tumor size criteria, which separate T1, T2, and T3 disease; and extension of base of tongue tumor onto the lingual surface of the epiglottis is still T3 disease, but T4a and T4b are combined into one as $\mathrm{T} 4$.

Nasopharyngeal Carcinoma. Nasopharyngeal carcinoma is most often nonkeratinizing differentiated or undifferentiated carcinoma, which is EBV-related and, in the United States, most often found in Asian populations, particularly those of southern Chinese migration or descent. HPV-related NPC and tobacco-/ alcohol-related EBV are less common worldwide. Staging is not currently altered by EBV status of the tumor; however, if EBVpositive nodal disease is found in the neck without a primary source determined, then the nodal metastases are presumed to be from the nasopharynx and they are designated T0 NPC and staged accordingly.

The most significant change to the T-assignment for NPC is the clarification of involvement of specific bony and masticator structures. Involvement of any bony structures such as cervical vertebrae, pterygoid plates, skull base, or paranasal sinuses is T3 disease. In the AJCC Cancer Staging Manual seventh edition, involvement of any masticator space structure was designated T4 disease. This has now been subdivided so that involvement of the medial or lateral pterygoid muscle or of the prevertebral muscles is T2 disease, but if there is extension of tumor beyond the lateral surface of the pterygoid muscle or involvement of the parotid gland, this is T4 disease (Fig 3 and Tables 3 and 6). This reflects study evidence of a relatively good prognosis for lateral pterygoid muscle infiltration compared with more extensive lateral tumor spread. $^{15}$

For NPC nodal disease, the clinical designation of N3 disease has been modified. In the seventh edition staging system, N3b disease was determined by the presence of $\geq 1$ supraclavicular node in the triangle of $\mathrm{Ho}$, with $\mathrm{N} 3 \mathrm{a}$ indicating any nodal mass measuring $>6 \mathrm{~cm}$ that lies above the supraclavicular fossa. From a radiologic perspective, a supraclavicular node was determined by the presence of a clavicle on the same axial imaging section; however, variable patient positioning in the scanner makes this designation unreliable, and for many radiologists, supraclavicular nodal status was left to the physical examination. N3 disease is now designated as any nodal mass of $>6 \mathrm{~cm}$ or any nodal mass below the inferior aspect of the cricoid - that is, any node that is level IV or Vb. There is no longer subdivision into N3a and N3b.

"Non-HPV OPSCC and Hypopharynx." This is an entirely new chapter after creation of distinct nasopharyngeal and HPV-related/p16+ oropharyngeal SCC chapters from a previous pharynx chapter that encompassed all pharyngeal tumors. There are, however, no changes to the T-staging described in the seventh edition for oropharyngeal and hypopharyngeal tumors. The nodal staging of these tumors will follow the non-HPV, non-EBV HN clinical $(\mathrm{cN})$ and pathologic $(\mathrm{pN})$ nodal staging definitions, with special attention to the new inclusion of ENE (Table 1).

Oral Cavity SCC. The chapter, historically entitled "Lip and Oral Cavity," will now be called "Oral Cavity," with the lip tumors now staged in the new chapter entitled "Cutaneous Carcinoma of HN."

In the seventh edition, one of the defining features requiring careful imaging evaluation of oral tongue tumors was the presence of invasion of the extrinsic tongue muscles. This feature has been difficult for pathologists to determine because it is typically not possible for them to distinguish intrinsic and extrinsic skeletal 

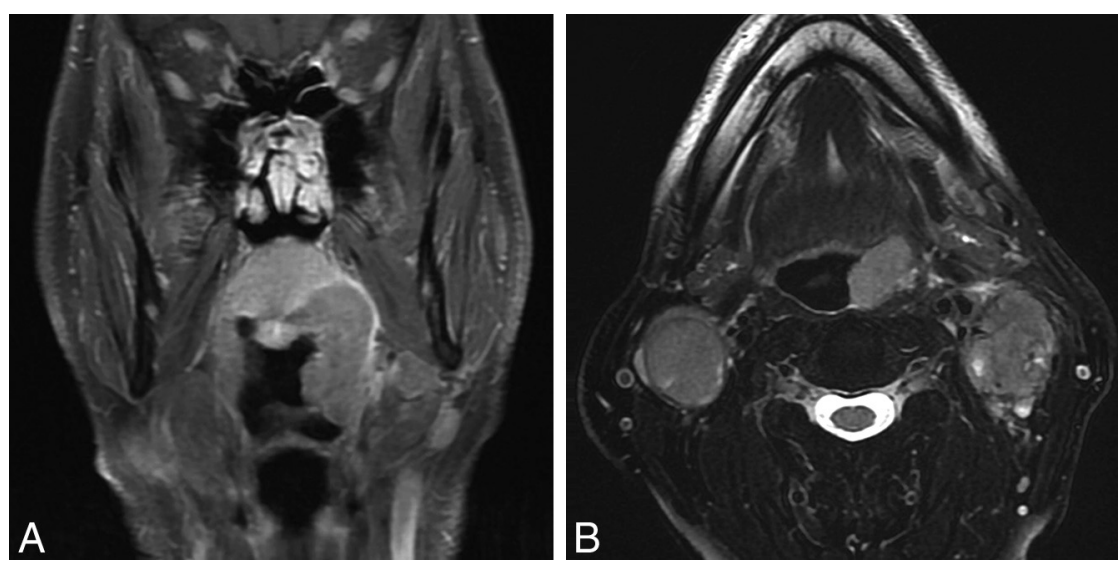

FIG 2. HPV-related p16(+) OPSCC in a 62-year-old man presenting with bilateral neck masses determined to be p16+ squamous cell carcinoma. Coronal T1 postcontrast fat-saturated MR image $(A)$ demonstrates a left tonsillar tumor extending superiorly to the soft palate. Because this tumor measured $>4 \mathrm{~cm}$ in longest dimension, it is assigned $\mathrm{T} 3$ with no change in the primary tumor staging from the seventh-to-eighth edition of the AJCC Cancer Staging Manual. Axial T2 fat-saturated image $(B)$ shows bilateral heterogeneous nodal masses with a high degree of suspicion of extranodal extension bilaterally. In the seventh edition, bilateral adenopathy of $<6 \mathrm{~cm}$ in greatest dimension for all OPSCCs is designated T2c disease, with prognostic grouping of T3N2c as stage IVA. In the new eighth edition, bilateral adenopathy of $<6 \mathrm{~cm}$ is N2 disease and the new prognostic grouping for p16 + OPSCC T3N2 is stage II. Extranodal extension does not affect the $\mathrm{N}$ designation for HPV/p16(+) OPSCC or NPC.

Table 4: Tumor staging for HPV-related OPSCC ${ }^{a}$

\begin{tabular}{ll}
\hline T Category & \multicolumn{1}{c}{ T Criteria } \\
\hline T0 & No primary identified \\
T1 & Tumor $\leq 2 \mathrm{~cm}$ in greatest dimension \\
T2 & Tumor $>2 \mathrm{but} \leq 4 \mathrm{~cm}$ in greatest dimension \\
T3 & Tumor $>4 \mathrm{~cm}$ in greatest dimension or extension \\
& to lingual surface of epiglottis \\
T4 & Moderately advanced local disease; tumor \\
& invades the larynx, extrinsic muscles of tongue, \\
& medial pterygoid, hard palate or mandible, or \\
& beyond \\
\hline
\end{tabular}

a The T4a and T4b features from OPSCC staging in the seventh edition are now combined as T4 in the new eighth edition. Table adapted with permission of the American Joint Committee on Cancer, Chicago, Illinois. The original source for this material is the AJCC Cancer Staging Manual.'

Table 5: AJCC clinical prognostic groupings for HPV-related OPSCC $^{\mathrm{a}}$

\begin{tabular}{llcc}
\hline When T Is... & And N Is... & $\begin{array}{c}\text { And } \\
\text { M Is... }\end{array}$ & $\begin{array}{c}\text { Then the Stage } \\
\text { Group Is... }\end{array}$ \\
\hline T0, T1, or T2 & N0 or N1 & M0 & I \\
T0, T1, or T2 & N2 & M0 & II \\
T3 & N0, N1, or N2 & M0 & II \\
Any T & N3 & M0 & III \\
T4 & Any N & M0 & III \\
Any T & Any N & M1 & IV \\
\hline
\end{tabular}

a Table adapted with permission of the American Joint Committee on Cancer, Chicago, Illinois. The original source for this material is the AJCC Cancer Staging Manual.'

muscles on sections. The eighth edition sees the removal of extrinsic muscle invasion as a T4 feature and the introduction of the depth of invasion (DOI) as a critical determinant of T-staging (Table 7). Depth of invasion is a pathology measurement and is the deep or endophytic invasive growth below a horizontal line drawn at the level of the basement membrane relative to the closest intact squamous mucosa. This is distinct from tumor thickness, which includes both the endophytic component and the exophytic tumor. Tumor infiltration below the basement membrane is the crit- ical factor for DOI and is very difficult to accurately assess by imaging. Measurement by the radiologist of tumor thickness may help guide the clinician in preoperative planning, but DOI is a pathologic assessment. It is still useful to comment on infiltration into the tongue or soft tissues of the oral cavity, particularly when evaluating the presence of bone invasion.

Nodal staging again follows the nodal groupings above, with separate nodal staging for HPV - and HPV + tumors and both clinical and pathologic nodal staging (Table 1). Radiologists should report nodal disease ipsilateral and contralateral to the primary site. Midline nodes in level IA, which are not uncommon with anterior oral cavity tumors, are considered ipsilateral to the primary tumor. Imaging should also evaluate ENE, though again this is ultimately a clinical examination or pathologic designation. Because neck dissections are frequently performed for oral cavity SCC, the $\mathrm{pN}$ tables for non-HPV SCC will be used for final staging designation.

"Cutaneous Carcinoma of HN." This entirely new chapter reorganizes all HN skin cancers other than eyelid SCC, melanoma, and Merkel cell carcinoma into a distinct chapter. Notably, lip SCC, which has traditionally been included with oral cavity tumor staging, is now included here. While tumor size constitutes much of the T-staging and is likely better determined by direct clinical examination, there are important features such as bone erosion, perineural tumor, and skull base invasion that should be sought by the radiologist when reading staging scans (Table 8). The nodal staging follows the $\mathrm{cN}$ and $\mathrm{pN}$ for all non-HPV, non-EBV SCCs elsewhere in the HN (Table 1).

\section{Key AJCC Changes without Specific Radiologic Impact}

Unknown Primary Carcinomas. Unknown primary carcinomas are characterized by the presentation of metastatic nodal disease in the absence of a defined primary tumor (T0). More than $90 \%$ of these tumors are HPV-related SCCs, and the occult primary is considered of oropharyngeal origin. ${ }^{16}$ When a primary tumor cannot be found by clinical examination or cross-sectional imaging, p16 mmunohistochemistry and HPV-ISH are performed. P16+ nodal disease should be further evaluated with HPV-ISH because p16+ (nonHPV-related) tumors can be found elsewhere in the HN. HPV and p16+ disease are likely of oropharyngeal origin and will therefore be categorized as T0. A positive EBV-ISH of nodal disease favors the unknown primary being of nasopharyngeal origin, T0.

SCC nodal disease that is p16-negative, HPV-negative, and $\mathrm{EBV}$-negative is not assigned to any primary site unless there is a known or suspected prior skin malignancy with the expected pattern of nodal disease. This metastatic nodal disease is staged using the $\mathrm{HN}$ clinical and pathologic nodal definitions for all non-HPV, 
non-EBV SCCs found in Chapter 6 of the AJCC Cancer Staging Manual (Fig 4 and Table 1).

Larynx. There are no changes to laryngeal T-staging, and again nodal staging follows the changes outlined previously for non-
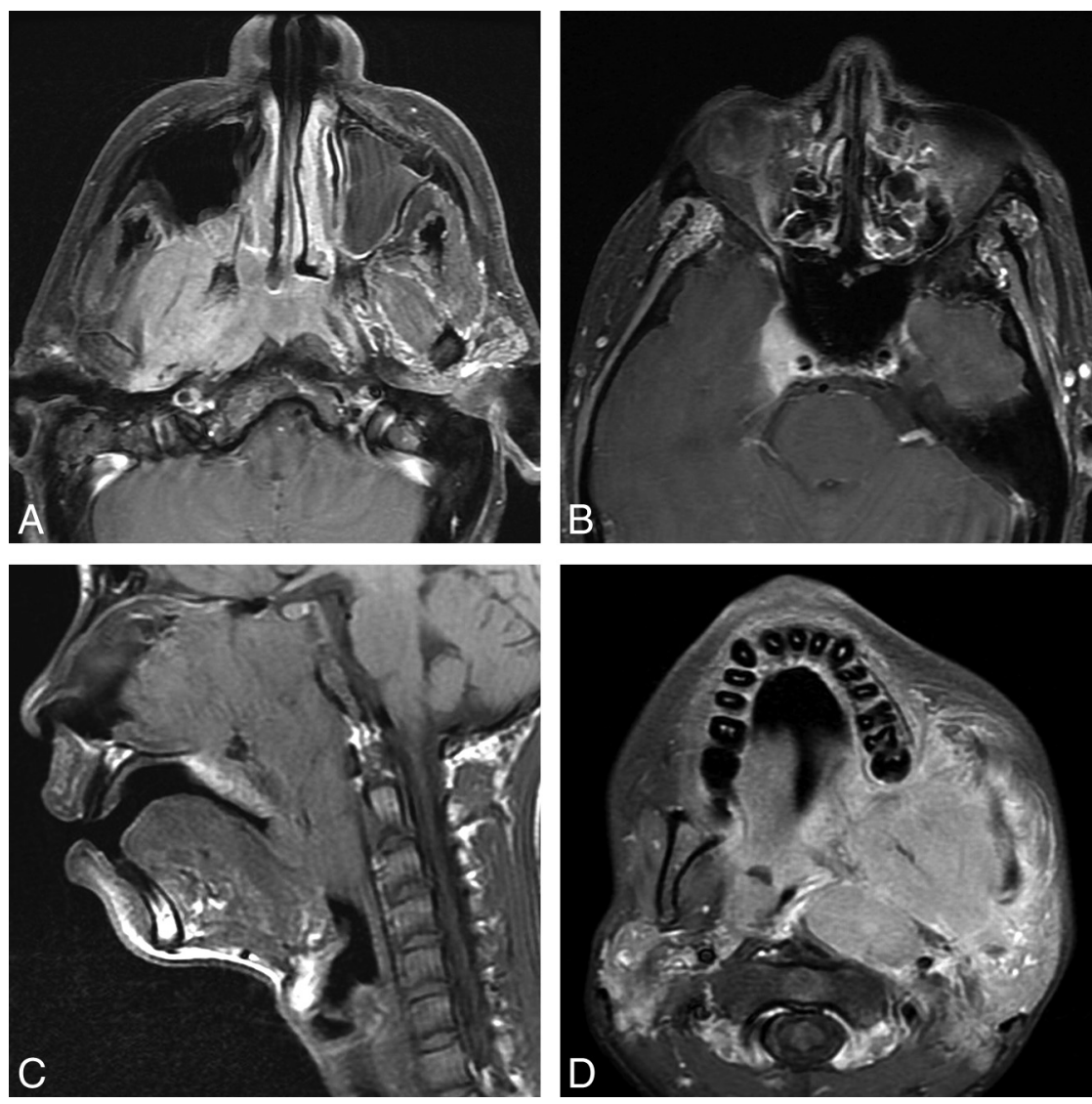

FIG 3. Two patients with nasopharyngeal carcinoma. $A$ and $B, A$ 64-year-old man with a nasopharyngeal mass found incidentally on PET/CT and determined to be EBV $(+)$ undifferentiated nonkeratinizing carcinoma. Axial T1 postcontrast fat-saturated images $(A$ and $B)$ demonstrate an asymmetric nasopharyngeal mass with lateral extension to the right masticator space lateral pterygoid muscle. In the seventh edition, this would be T4 disease; however, in the eighth edition, this is only T2 disease. Note however that in this patient, there is also involvement of the pterygoid plates, which is now clarified as T3 disease, and superior extension to the right cavernous sinus, which is T4 disease in both AJCC Cancer Staging Manual seventh and eighth editions. $C$ and $D, A 15$-year-old boy presenting with epistaxis and a large mass arising from the nasopharynx determined to be $E B V(+)$ undifferentiated nonkeratinizing carcinoma. Sagittal Tl (C) shows the large mass filling the nasopharynx, extending inferiorly to the oropharynx and anteriorly to the nasal cavity. Axial postcontrast $T 7$ with fat saturation $(D)$ shows lateral extension of the mass into the masticator space but also to the left parotid gland. In the eighth edition, involvement of the parotid gland or extension of tumor beyond the lateral surface of the lateral pterygoid muscle determines T4 status.
EBV, non-HPV SCCs for the entire HN (Table 1). Unknown primary tumors that are EBV and HPV negative are not assigned to the larynx as outlined above. When one evaluates primary laryngeal tumors, imaging offers utility for evidence of invasion of the paraglottic space (T3) and inner lamina of the thyroid cartilage (T3) or invasion through the outer thyroid cortex or of the cricoid (T4a). Arytenoid and epiglottic cartilage involvement does not change the laryngeal tumor stage.

Major Salivary Gland Tumors. There have been no changes to the T-staging tables for salivary masses. The N nodal staging table changes reflect those described above for all p16-, EBV - HN nodal disease (Table 1). Again, SCC masses in the parotid gland may reflect metastatic nodal disease, particularly from cutaneous facial and scalp malignancies.

Nasal Cavity and Paranasal Sinuses. There have been no significant changes to the T-staging for nasal cavity and paranasal sinuses, and the N nodal staging tables reflect those described above for the HN (Table 1). Both CT and MR imaging offer advantages for complete pretreatment evaluation of sinonasal malignancies and are often both used. CT offers superior detail for hard palate invasion, while MR imaging allows better discrimination of tumor from obstructed sinus secretions. MR imaging offers a more detailed evaluation for dural or brain parenchymal involvement as well as perineural tumor spread. Both allow nodal evaluation with the caveat that retropharyngeal nodal tumor may be more conspicuous on MR imaging when isodense to prevertebral muscles on contrast-enhanced CT.

Mucosal Melanoma of the HN. There are no changes to this staging system for mucosal melanoma of the $\mathrm{HN}$ com-

Table 6: Tumor definitions for nasopharyngeal carcinoma ${ }^{a}$

\begin{tabular}{ll}
\hline T Category & T Criteria \\
\hline Tx & Primary tumor cannot be assessed \\
T0 & No tumor identified, but EBV + cervical lymph node(s) involvement \\
Tis & In situ carcinoma \\
T1 & Tumor confined to nasopharynx, or extension to oropharynx and/or nasal cavity without parapharyngeal involvement \\
T2 & Tumor with extension to parapharyngeal space, and/or adjacent soft-tissue involvement (medial pterygoid, lateral \\
& pterygoid, prevertebral muscles) \\
T3 & Tumor with infiltration of bony structures at skull base, cervical vertebrae, pterygoid structures, and/or paranasal sinuses \\
T4 & Tumor with intracranial extension, involvement of cranial nerves, hypopharynx, orbit, parotid gland, and/or extensive \\
& soft-tissue infiltration beyond the lateral surface of the lateral pterygoid muscle \\
\hline
\end{tabular}

a The eighth edition shows critical changes from the prior edition with downstaging of masticator space involvement from T4 to T2 and clarification of the T-staging for invasion of bony structures. Table adapted with permission of the American Joint Committee on Cancer, Chicago, Illinois. The original source for this material is the AJCC Cancer Staging Manual. 


\begin{tabular}{ll}
\hline T Category & T Criteria \\
\hline Tx & Primary tumor cannot be assessed \\
Tis & Carcinoma in situ \\
T1 & Tumor $\leq 2 \mathrm{~cm}, \leq 5-\mathrm{mm} \mathrm{DOI}$; DOI is depth of invasion and not tumor thickness \\
T2 & Tumor $\leq 2 \mathrm{~cm}, \mathrm{DOI}>5 \mathrm{~mm}$ and $\leq 10 \mathrm{~mm}$ or tumor $>2$ but $\leq 4-\mathrm{cm}$ and $\leq 10-\mathrm{mm} \mathrm{DOI}$ \\
T3 & Tumor $>4 \mathrm{~cm}$ or any tumor $>10-\mathrm{mm}$ DOI \\
T4a & Moderately advanced local disease; tumor invades adjacent structures only (eg, through cortical bone of the mandible or \\
& maxilla or involves the maxillary sinus or skin of the face); note that superficial erosion of bone/tooth socket (alone) by \\
& a gingival primary is not sufficient to classify a tumor as T4 \\
T4b & Very advanced local disease; tumor invades masticator space, pterygoid plates, or skull base and/or encases the internal \\
& carotid artery
\end{tabular}

${ }^{a}$ Note that the greatest diameter of the primary tumor is still measured, but primary tumor depth of invasion is also a critical determinant of T-staging. This is the deep extent of tumor invasion below the surface and is distinct from tumor thickness. Lip tumors are no longer staged along with oral cavity SCC, but using the tables from "Cutaneous Carcinoma of HN." Table adapted with permission of the American Joint Committee on Cancer, Chicago, Illinois. The original source for this material is the AJCC Cancer Staging Manual.

Table 8: Tumor definitions for cutaneous carcinoma of the $\mathbf{H N}^{\mathbf{a}}$

\begin{tabular}{ll}
\hline T Category & \multicolumn{1}{c}{ T Criteria } \\
\hline Tx & Primary tumor cannot be identified \\
Tis & Carcinoma in situ \\
T1 & Tumor $\leq 2 \mathrm{~cm}$ in greatest dimension \\
T2 & Tumor $>2$ but $\leq 4 \mathrm{~cm}$ in greatest dimension \\
T3 & Tumor $>4 \mathrm{~cm}$ in maximum dimension or minor bone \\
& $\quad$ erosion or perineural invasion, or deep invasion ${ }^{\mathrm{b}}$ \\
T4a & Tumor with gross cortical bone/marrow invasion \\
T4b & Tumor with skull base invasion and/or skull base \\
& foramen involvement \\
\hline
\end{tabular}

a This new chapter in the eighth edition should be used for all HN cutaneous malignancies except the eyelid and cutaneous melanoma and Merkel cell carcinoma. Lip tumors are now staged using this table. Table adapted with permission of the American Joint Committee on Cancer, Chicago, Illinois. The original source for this material is the AJCC Cancer Staging Manual.'

${ }^{b}$ Deep invasion is defined as invasion beyond the subcutaneous fat or $>6 \mathrm{~mm}$ (as measured from the granular layer of adjacent normal epidermis to the base of the tumor). Perineural invasion for the T3 classification is defined as tumor cells within the nerve sheath of a nerve lying deeper than the dermis or measuring $\geq 0.1 \mathrm{~mm}$ in caliber or presenting with clinical or radiographic involvement of named nerves without skull base invasion or transgression.

pared with the seventh edition. As a reminder for radiologists when evaluating these aggressive tumors, there is only $\mathrm{T} 3$ or $\mathrm{T} 4 \mathrm{a} / \mathrm{b}$ disease (there is no T1 or T2). There is also a tumor-specific mucosal melanoma nodal staging in which N0 is the absence of nodal metastasis and $\mathrm{N} 1$ is the presence of regional nodal disease. Nx is used when regional nodes cannot be assessed. Extranodal extension has not been added to this nodal staging. There is not yet a prognostic stage grouping for mucosal melanoma.

\section{Final Reminders about Staging}

1) For all sites in the head and neck, M0 is used when there is a negative metastatic work-up or there are no known metastases. M1 is the presence of metastatic disease. There is no Mx designation.

2) When in doubt, the general rules of AJCC staging are to assign the lower of the two T, N, or M categories-that is, to downstage rather than upstage. ${ }^{17}$

3) Tumor staging is a team effort. The radiologist can add significant value to tumor boards by attending them, explaining the radiologic subtleties, and being aware of the critical features that distinguish different $\mathrm{T}$ and $\mathrm{N}$ categories.

\section{Summary}

Perhaps the most dramatic changes noticeable to radiologists who are participating in tumor boards or who have been making it a

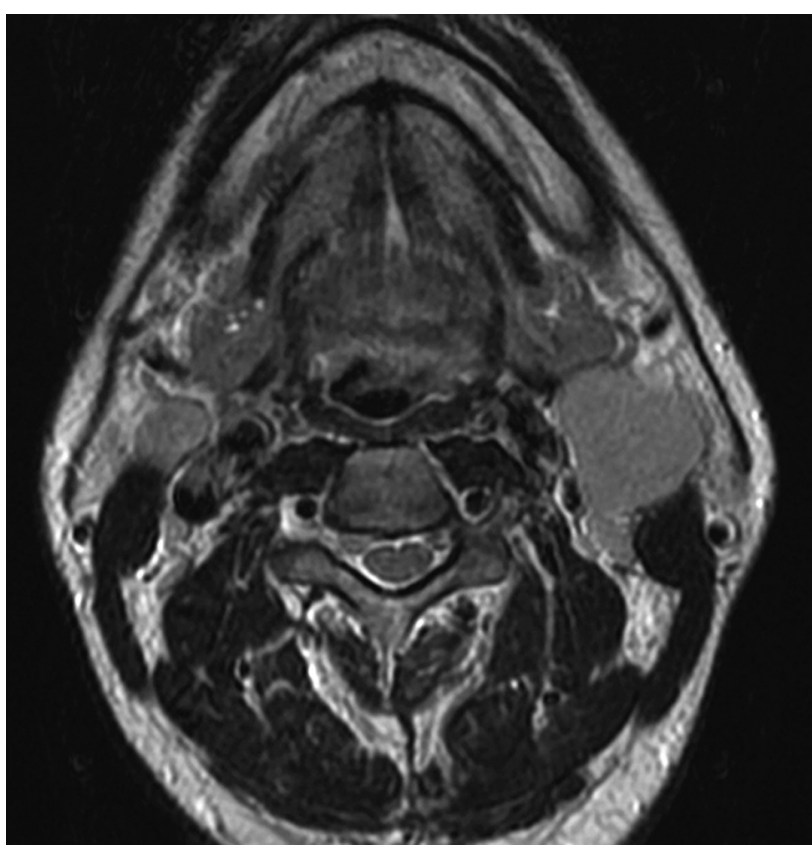

FIG 4. Axial T2-weighted MR image through the neck in a 45-year-old man presenting with neck masses. The enlarged solid left level 2A node measured $3.5 \mathrm{~cm}$ in maximal diameter with additional abnormal nodes seen on the right at $2 \mathrm{~A}$ and bilaterally at levels 3 and $5 \mathrm{~A}$. No primary tumor was evident on clinical examination or on this MR imaging. In the absence of a primary tumor, it is difficult to determine which nodal table to use, though most unknown primary tumors are $\mathrm{HPV} / \mathrm{pl6}(+)$ OPSCC. If fine-needle aspiration of the node reveals $\mathrm{HPV} / \mathrm{p} 16(+)$, then an OPSCC primary would be assumed and this would be assigned TON2. If pathology revealed HPV-negative, p16 negative, and ENB negative, then this would be assigned N2c and no primary site assigned. Fine-needle aspiration revealed this to be undifferentiated, nonkeratinizing carcinoma $\mathrm{EBV}+$, with categories then assigned as TON2 NPC.

practice to include TNM categories in the radiology report are those relating to HPV-related p16+ OPSCC and nodal staging. There is a dramatic downstaging of HPV-related p16(+) OPSCC disease with the new staging system, reflecting the overall markedly better prognosis of these tumors compared with p16(-) OPSCC. Because p16/HPV status may be unavailable to the radiologist at the time of reporting, it remains important, as with all reports for tumor staging, to include all the relevant imaging information of tumor size and extent of invasion. This information can then be combined with immunohistochemistry to determine the accurate $\mathrm{p} 16(+)$ or $\mathrm{p} 16(-)$ OPSCC clinical stage. 
Nodal staging has also expanded so that there are different clinical nodal categories (cN) for HPV/p16(+) OPSCC, EBV $(+)$ NPC, and all other HN SCCs. There are also 2 new pathologic nodal staging systems $(\mathrm{pN})$ for HPV/p16(+) OPSCC and all other HN SCCs when neck dissections are performed. The standard imaging-based classification should be used to describe nodal levels, and it is important that radiologists clarify both ipsilateral and contralateral nodes that appear positive and whether there is perinodal infiltration of adjacent fat or muscles, the strongest imaging features supporting ENE. Currently the staging designation of $\mathrm{ENE}+$ requires gross clinical examination evidence of ENE or pathologic demonstration of ENE, but it will be very useful for radiologists to describe features suspicion for ENE so that the clinician can re-evaluate the clinical nodal status.

Additional, less complex changes have been made to the staging in other $\mathrm{HN}$ sections to reflect the changing understanding of tumor biology and pathophysiology and patient outcomes. Radiologists are encouraged to become familiar with the new TNM system when reporting staging scans and to include all relevant imaging information in reports to facilitate cancer care. Regardless of whether an individual radiologist includes TNM categories in their report, it is important that we try to understand the process of tumor staging, the critical role radiologists play in providing staging information, and the value we add to patient care. The AJCC and UICC eighth editions will be implemented for staging new tumors as of January 1, 2018.

Disclosures: Christine M. Glastonbury-RELATED: Support for Travel to Meetings for the Study or Other Purposes: all authors reimbursed for travel for a AJCC committee meeting. William M. Lydiatt-UNRELATED: Royalties: Up-to-Date and Cancers of the Mouth and Throat.

\section{REFERENCES}

1. Amin MB, Edge SB, Greene FL, et al, eds. AJCC Cancer Staging Manual. 8th ed. New York: Springer-Verlag; 2017

2. Brierley JD, Gospodarowicz MK, Wittekind C, eds, TNM Classification of Malignant Tumours. Hoboken: Wiley Blackwell; 2017

3. Edge S, Byrd DR, Compton CC, et al, eds. AJCC Cancer Staging Manual. 7th ed. New York: Springer-Verlag; 2010

4. Ang KK, Harris J, Wheeler R, et al. Human papillomavirus and survival of patients with oropharyngeal cancer. N Engl J Med 2010;363: 24-35 CrossRef Medline

5. O'Sullivan B, Huang SH, Su J, et al. Development and validation of a staging system for HPV-related oropharyngeal cancer by the International Collaboration on Oropharyngeal Cancer Network for Staging (ICON-S): a multicentre cohort study. Lancet Oncol 2016; 17:440-51 CrossRef Medline

6. Huang SH, Xu W, Waldron J, et al. Refining American Joint Committee on Cancer/Union for International Cancer Control TNM stage and prognostic groups for human papillomavirus-related oropharyngeal carcinomas. J Clin Oncol 2015;33:836-45 CrossRef Medline

7. Haughey BH, Sinha P, Kallogjeri D, et al. Pathology-based staging for HPV-positive squamous carcinoma of the oropharynx. Oral Oncol 2016;62:11-19 CrossRef Medline

8. Wreesmann VB, Katabi N, Palmer FL, et al. Influence of extracapsular nodal spread extent on prognosis of oral squamous cell carcinoma. Head Neck 2016;38(suppl 1):E1192-99 CrossRef Medline

9. Myers JN, Greenberg JS, Mo V, et al. Extracapsular spread: a significant predictor of treatment failure in patients with squamous cell carcinoma of the tongue. Cancer 2001;92:3030-36 Medline

10. Sinha P, Lewis JS Jr, Piccirillo JF, et al. Extracapsular spread and adjuvant therapy in human papillomavirus-related, p16-positive oropharyngeal carcinoma. Cancer 2012;118:3519-30 CrossRef Medline

11. Lydiatt WM, Patel SG, O’Sullivan B, et al. Head and neck cancersmajor changes in the American Joint Committee on cancer eighth edition cancer staging manual. CA Cancer J Clin 2017;67:122-37 CrossRef Medline

12. King AD, Tse GM, Yuen EH, et al. Comparison of CT and MR imaging for the detection of extranodal neoplastic spread in metastatic neck nodes. Eur J Radiol 2004;52:264-70 CrossRef Medline

13. Lodder WL, Lange CA, van Velthuysen ML, et al. Can extranodal spread in head and neck cancer be detected on MR imaging. Oral Oncol 2013;49:626-33 CrossRef Medline

14. Prabhu RS, Magliocca KR, Hanasoge S, et al. Accuracy of computed tomography for predicting pathologic nodal extracapsular extension in patients with head-and-neck cancer undergoing initial surgical resection. Int J Radiat Oncol Biol Phys 2014;88:122-29 CrossRef Medline

15. Pan JJ, Ng WT, Zong JF, et al. Prognostic nomogram for refining the prognostication of the proposed 8th edition of the AJCC/UICC staging system for nasopharyngeal cancer in the era of intensitymodulated radiotherapy. Cancer 2016;122:3307-15 CrossRef Medline

16. Motz K, Qualliotine JR, Rettig E, et al. Changes in unknown primary squamous cell carcinoma of the head and neck at initial presentation in the era of human papillomavirus. JAMA Otolaryngol Head Neck Surg 2016;142:223-28 CrossRef Medline

17. Gress DM, Edge SB, Greene FL, et al. Principles of cancer staging. In: Amin MB, Edge SB, Greene FL, et al, eds. AJCC Cancer Staging Manual. 8th Ed. New York: Springer-Verlag; 2017:3-30 